\title{
Constantes en los movimientos estudiantiles latinoamericanos: Aproximación a partir del caso chileno de $2011^{1}$
}

\author{
Constant features in Latin American student movements: an approach \\ based on the Chilean case of 2011
}

Firmes nos movimentos estudantis latino-americanos: aproximação a partir do caso chileno de 2011

Andrés Donoso Romo ${ }^{2}$

Universidad de Playa Ancha (Chile)

Recepción: 12/07/2016

Evaluación: 12/04/2017

Aceptación: 05/05/2017

Artículo de Revisión

DOI: https://doi.org/10.19053/01227238.6163

\section{RESUMEN}

En el siguiente artículo se identifican de las demandas y de los logros del una serie de similitudes, tanto formales como sustanciales, entre los principales movimientos estudiantiles ocurridos en América Latina en los últimos cien años: el chileno de 2011, el mexicano de 1968, el brasileño de 1968 y el argentino de 1918. Objetivo que se alcanza utilizando como columna vertebral del trabajo la descripción de los rasgos fundamentales, movimiento chileno de 2011, los cuales van siendo contrastados, inmediatamente, con lo sucedido en los otros tres movimientos considerados. Un ejercicio que, además de ser original por cuanto la mayoría de los estudios dedicados a este tipo de fenómenos se circunscriben a casos nacionales, ve incrementada su importancia al constatar que en 2018

$1 \quad$ El artículo informa parte de los resultados del proyecto CONICYT/FONDECYT Concurso de Iniciación $\mathrm{N}^{\circ} 11140250$. Una versión preliminar de este trabajo fue expuesta en la conferencia "Neoliberalismo cuestionado: aproximación al movimiento estudiantil chileno de 2011”, dictada en el Instituto de Investigaciones sobre la Universidad y la Educación de la Universidad Nacional Autónoma de México, el 9 de septiembre de 2015.

2 Investigador del Centro de Estudios Avanzados de la Universidad de Playa Ancha, Valparaíso, Chile. Doctor en Integración en América Latina por la Universidade de São Paulo. Maestro en Estudios Latinoamericanos por la Universidad de Chile. Email: andres.donoso@upla.cl 
se conmemorarán simultáneamente varias fechas significativas en la materia, celebraciones donde serán muy bien recibidas interpretaciones integradoras como las que aquí se presentan. Es necesario precisar que metodológicamente el artículo se sustenta en un proceso sistemático de análisis de contenido, labor que fue facilitada por un programa computacional afín y donde se incluyeron los textos claves que analizan cada uno de estos movimientos. Estrategia que permitió concluir, efectivamente, que estos movimientos poseen un conjunto de características compartidas como, por ejemplo, el que usaran similares repertorios de acción o que evidenciaran una marcada preocupación por asuntos extra-universitarios.

Palabras clave: Revista Historia de la Educación Latinoamericana, Estudiante, Movimiento estudiantil, Chile, América Latina, Historia contemporánea.

\section{ABSTRACT}

This paper identifies some constant features, both formal and substantial, among the major student movements in Latin America over the last hundred years: the Chilean in 2011, the Mexican in 1968, the Brazilian in 1968, and the Argentinian in 1918. The objective of this study is accomplished by using as a backbone the description of the fundamental features regarding the demands and achievements of the Chilean movement of 2011, which are contrasted with the events taking place in the other three movements. This exercise, in addition to being original, because most studies dedicated to this type of phenomena are confined to national cases, has increased its importance in finding that in 2018 will be simultaneously commemorated several significant dates. Within these celebrations, integrative interpretations such the ones presented here, will be very well received. It is necessary to specify that this article is methodologically based on a systematic process of content analysis, which was facilitated by a software where the key texts that analyze these movements were included. This strategy led to the conclusion that these movements have a set of shared features, such as the use of similar repertoires of action or the fact that they evidenced a strong concern for extra-university issues. Keywords: History of Latin American Education, Student, Student Movement, Chile, Latin America, Contemporary History.

\section{RESUMO}

No seguinte artigo se identificam uma série de similitudes, tanto formais como substanciais, entre os principais movimentos estudantis ocorridos na América Latina nos últimos cem anos: o chileno de 2011, o mexicano de 1968, o brasileiro de 1968 e o argentino de 1918. Este objetivo foi alcançado se utilizando como coluna vertebral do trabalho a descrição dos traços fundamentais das demandas e dos sucessos do movimento chileno de 2011, os quais vêm sendo contrastados, imediatamente, com o ocorrido nos outros três movimentos considerados. Um exercício que, apesar de ser original, uma vez que a maioria dos estudos dedicados a este tipo de fenômenos se circunscrevem a casos nacionais, vê incrementada sua 
importância ao constatar que em 2018 se comemorariam simultaneamente varias datas significativas dentro dessa matéria, celebrações nas quais serão muito bem recebidas interpretações integradoras com as que aqui se apresentam. É necessário enfatizar que, metodologicamente, o artigo se sustenta num processo sistemático de análise de conteúdo, esforço que foi facilitado por um programa computacional afim, onde se incluíram os textos chave que analisam cada um destes movimentos. Estratégia que permitiu concluir, efetivamente, que estes movimentos possuem um conjunto de características compartilhadas como, por exemplo, os que usaram repertórios similares de ação ou que evidenciam uma clara preocupação por assuntos extrauniverstários.

Palavras-chave: Revista História da Educação Latino-americana, Estudante, Movimento estudantil, Chile, América Latina, História contemporânea.

\section{INTRODUCCIÓN}

Previendo las múltiples conmemoraciones que en 2018 celebrarán los cincuenta años de los movimientos estudiantiles de 1968 -entre ellos el brasileño, chileno, mexicano y uruguayo- y que rememorarán también los cien años del movimiento argentino de 1918 -y sus desdoblamientos en Chile, Colombia, Cuba, México y Perú-, en este artículo se entregarán algunos insumos para facilitar la tarea de pensar estos fenómenos en conjunto. Tarea que incrementa su relevancia si se considera que el grueso de las investigaciones que se han dedicado a analizar este tipo de movimientos tienden a circunscribirse a casos puntuales ${ }^{3}$.

A sabiendas de que son muchos los caminos para pensar los movimientos estudiantiles latinoamericanos como un todo, y sin el ánimo de menospreciar otros posibles abordajes, aquí se ha optado por utilizar al movimiento estudiantil chileno de 2011 como eje estructural de los análisis y por emplear referencias a otros grandes alzamientos juveniles sucedidos en la región durante el siglo XX para identificar posibles constantes entre ellos. Los alzamientos estudiantiles que servirán para contrastar al movimiento chileno serán, más puntualmente, el sucedido en México en 1968, el de Brasil en 1968 y el de Argentina en 19184. Ante la posibilidad de que algún lector no esté familiarizado con los movimientos aquí considerados éstos se bosquejarán brevemente.

3 Balance que puede ser refrendado al revisar los trabajos que componen la principal fuente de estudio de estos fenómenos en la región, los libros que desde 1999 viene coordinando la investigadora Renate Marsiske y que llevan por título Movimientos estudiantiles en América Latina. Cabe consignar, a su vez, que hay dos obras que funcionan como excepciones que confirman la regla, pues tienen una profunda vocación latinoamericana en sus análisis, ellas son: Renate Marsiske, Movimientos estudiantiles en América Latina: Argentina, Perú, Cuba y México, 1918-1929 (Ciudad de México: Centro de Estudios sobre la Universidad de la Universidad Nacional Autónoma de México, 1989) y Juan Carlos Portantiero, Estudiantes y política en América Latina: el proceso de la reforma universitaria (1918-1930) (Ciudad de México: Siglo XXI, 1987).

4 Conscientes de que la lista de grandes movimientos estudiantiles supera largamente los que aquí se están considerando, se precisa que estos han sido escogidos por dos razones. Primero, porque efectivamente han sido algunos de los más multitudinarios y prolongados que ha conocido la región. Y segundo, porque han sido muy bien estudiados, siendo sobre esos estudios que se generarán las interpretaciones que aquí se presentarán. 
El movimiento chileno de 2011 se prolongó por poco más de cinco meses, fue liderado por el estudiantado universitario e involucró a cientos de miles de manifestantes provenientes del mundo cultural y educacional del país. Sus principales demandas, motivadas por la asfixia económica que vivía el estudiantado por causa de los altos aranceles que debían pagar, fueron gratuidad en la educación superior y fin al lucro en las instituciones educacionales. Dos exigencias que, dicho sea de paso, tuvieron tal fuerza que inclusive lograron poner en entredicho la arquitectura educacional impuesta durante la última dictadura.

El movimiento mexicano de 1968, desarrollado durante el segundo semestre de ese año, involucró al estudiantado de decenas de instituciones de educación superior del país y, entre ellas, al de las dos más numerosas, la Universidad Nacional Autónoma de México y el Instituto Politécnico Nacional. Su principal demanda fue poner fin al régimen político autoritario que imperaba en el país y es recordado, preferentemente, por la matanza que selló su suerte aquel fatídico 2 de octubre ${ }^{5}$.

El movimiento brasileño de 1968, en tanto, comenzó a fines de marzo de ese año en el marco de las conmemoraciones de un aniversario más del golpe de Estado que sufriera el país en 1964. La muerte de un estudiante a manos de la policía hizo que el estudiantado de las principales ciudades del país se levantara contra el régimen dictatorial. A esta primera ola de protesta le siguió una segunda, en junio, que será al mismo tiempo su apogeo, y una tercera ola, su epílogo, en octubre, fecha que coincide con el encarcelamiento de más de setecientos dirigentes estudiantiles que participaban en un congreso clandestino ${ }^{6}$.

El movimiento universitario argentino de 1918, probablemente uno de los más conocidos por sus múltiples desdoblamientos en el resto de América Latina, se inició a fines de 1917 en la Universidad de Córdoba por problemas académicos que afectaban al estudiantado de medicina e ingeniería. Luego de un año, previa incorporación de los estudiantes de las otras cuatro casas de estudios superiores que funcionaban en el país en ese entonces, el movimiento terminó con un sonado triunfo que se plasmó en la inclusión de los estudiantes en la estructura de gobierno universitario ${ }^{7}$.

Con el espíritu de favorecer el estudio de estas páginas se ha juzgado conveniente transparentar los tres supuestos sobre las cuales ellas descansan. Primero, se asume que la historia contemporánea de América Latina comienza a fines del

5 Revisar, entre otros textos, Alberto del Castillo Troncoso, Ensayo sobre el movimiento estudiantil de 1968: la fotografía y la construcción de un imaginario (Ciudad de México: Instituto Mora - Instituto de Investigaciones sobre la Universidad y la Educación de la Universidad Nacional Autónoma de México, 2012); Ramón Ramírez, El movimiento estudiantil de México (julio/diciembre de 1968) (Ciudad de México: Era y Benemérita Universidad Autónoma de Puebla, 2008); y Sergio Zermeño, México una democracia utópica: el movimiento estudiantil del 68 (Ciudad de México: Siglo XXI, 2010).

6 Revisar, entre otros textos, Artur Jose Poerner, O poder jovem: história da participação política dos estudantes brasileiros (San Pablo: Centro de Memória da Juventude, 1995); Mario Ribeiro do Valle, 1968, o diálogo é a violência: movimento estudantil e ditadura militar no Brasil (Campinas: Editora Unicamp, 2010); y José Luis Sanfelice, Movimento estudantil, a UNE na resistência ao golpe de 1964 (Campinas: Editora Alínea, 2008).

7 Revisar, entre otros textos, Pablo Buchbinder, ¿Revolución en los claustros? La Reforma Universitaria de 1918 (Buenos Aires: Editorial Sudamericana, 2008); Alberto Ciria y Horacio Sanguinetti, La reforma universitaria (1918-2006) (Santa Fe: Universidad Nacional del Litoral, 2006); y Roberto Ferrero, Historia crítica del movimiento estudiantil de Córdoba, Tomo 1 (1918-1943) (Córdoba: Alción, 1999). 
siglo XIX. Un deslinde que se ha escogido porque es entonces que comienzan a verificarse dos fenómenos que modifican dramáticamente el diario vivir de la población, y que todavía continúan dando su sello a nuestro tiempo, la industrialización y la urbanización. Cambios que transformaron los modos de vivir y de trabajar de las personas y también, ciertamente, las formas de articulación política y las configuraciones culturales ${ }^{8}$. No está demás subrayar que para los fines perseguidos en este artículo lo relevante de situar el inicio de la época actual a fines del siglo XIX es que permite comprender que algunos acontecimientos que a simple vista parecieran estar disociados, como el movimiento estudiantil argentino de 1918 y el movimiento chileno de 2011, forman parte de un mismo momento histórico.

Segundo, se observa a las protestas estudiantiles como fenómenos sociales persistentes que en ciertos momentos, como en el año 2011 en Chile, alcanzan un máximo de intensidad que les permite convertirse en un movimiento social propiamente tal, para luego entrar en fases de repliegue, latencia y/o acumulación de fuerzas ${ }^{9}$. Lo que implica comprender que los movimientos estudiantiles no serían fenómenos aislados, azarosos o excepcionales, como tienden a juzgarlos los analistas que no poseen formación histórica, sino que serían, mas bien, malestares acumulados a lo largo de años, incluso décadas, que debido a la conjunción de condicionantes coyunturales, circunstanciales y/o estructurales se expresarían impetuosamente en un período acotado de tiempo. Presunción que permite comprender, a su vez, que aún cuando el agravio que haga detonar un movimiento pueda ser algo puntual, y a veces un detalle, este será capaz de desencadenar distintas demandas acumuladas o, mejor dicho, no resueltas en protestas precedentes.

Tercero, se entiende que los grandes movimientos estudiantiles contemporáneos en América Latina, a diferencia de sus expresiones precedentes, no solo se erigen alrededor de aspectos gremiales o educacionales, también levantaron cuestionamientos al orden político o social en que se enmarcan. De hecho, aunque fue a fines del siglo XVIII, con los primeros destellos independentistas en la región, que entre los universitarios se comienza a demandar una educación diferente, útil, acorde a las nuevas ideas de progreso surgidas de la primera revolución industrial, es solo con los cambios económicos y sociales reseñados para finales del siglo XIX, con la aparición de los sectores medios urbanos y los graves problemas que comienzan a castigar a los sectores populares, que el estudiantado empieza a hacer de la "cuestión social" un motivo de sus preocupaciones.

$8 \quad$ Entre los intelectuales que coinciden con este deslinde temporal se cuentan Pablo González Casanova y Roberto Fernández Retamar. Revisar, por ejemplo, Pablo González Casanova, Imperialismo y liberación: una introducción a la historia contemporánea de América Latina (Ciudad de México: Siglo XXI, 1985), 11; y Roberto Fernández Retamar, Pensamiento de Nuestra América (Buenos Aires: Consejo Latinoamericano de Ciencias Sociales, 2006), 39. En un trabajo anterior también hemos profundizado en esta materia, revisar Andrés Donoso Romo, Identidad y educación en América Latina. Ensayos (Caracas: Laboratorio Educativo, 2012), 13-28.

9 Supuesto que también es compartido por Camila Vallejo, Podemos cambiar el mundo (Santiago de Chile: Ocean Sur, 2012), 4748. Quien desee profundizar en el ciclo de manifestaciones en donde se insertó el movimiento estudiantil chileno de 2011 puede consultar Giorgio Jackson, El país que soñamos (Santiago de Chile: Random House Mondadori, 2013), 55; Daniel Núñez, "Proyecciones políticas del movimiento social por la educación en Chile", Revista del Observatorio Social de América Latina, $\mathrm{n}^{\circ} 31$ (2012): 63; y Manuel Riesco, Parto de un siglo: una mirada al mundo desde la izquierda de América Latina (Santiago de Chile: Centro de Estudios Nacionales de Desarrollo Alternativo - Editorial Universidad de Santiago de Chile, 2012), 244-245. 
Dicho con otras palabras, cuando la universidad comienza a ser apropiada por los sectores medios, cuando deja de ser un reducto exclusivo de la oligarquía, de esos herederos con un porvenir económico resuelto, el estudiantado empieza a mirar más allá de los límites de la institución para imaginar escenarios políticos-sociales que garanticen un mejor pasar para ellos y también para las grandes mayorías que los ayudarían a lograrlos ${ }^{10}$.

Descritos los propósitos del artículo, subrayada su importancia, bosquejados los movimientos que complementarán los análisis y transparentados los supuestos que tornan inteligibles las interpretaciones a presentar, solo resta agregar que a esta introducción le sucederán tres secciones y que todas se valdrán de un mismo eje narrativo: primero se detallará lo referido al movimiento chileno de 2011 e inmediatamente se evaluará la persistencia histórica de dicho elemento en los otros tres movimientos considerados. Conforme lo señalado, la siguiente sección profundiza en los rasgos fundamentales del movimiento estudiantil chileno de 2011 e identifica su existencia en los otros movimientos estudiados. Luego le sigue una sección donde se ahonda en las principales demandas que enarboló el levantamiento chileno de 2011 al tiempo que se establecen algunos puentes interpretativos con las exigencias levantadas en los otros casos cotejados. La última sección, en tanto, se concentra en el desenlace y los logros del movimiento chileno de 2011 vistos a la luz del epílogo y las conquistas de los otros movimientos considerados.

\section{Rasgos fundamentales del movimiento estudiantil chileno de 2011 en clave latinoamericana}

Los rasgos fundamentales del movimiento estudiantil chileno de 2011, aquellos aspectos que tienen que ver con los elementos que lo desencadenaron, con su masividad, con los actores que lo conformaron y con la estructura organizacional que lo condujo, serán reseñados y contrastados, inmediatamente, con aquellos aspectos que este movimiento compartió con los vivenciados en México en 1968, en Brasil en 1968 y en Argentina en 1918.

Los hechos que dieron inicio al movimiento estudiantil chileno de 2011, como ha sido una constante en todos los movimientos de estas dimensiones, pueden ser vistos como nimiedades. La falta de reajuste en los montos de unas becas de alimentación que beneficiaban a parte de los estudiantes más pobres, sumados a una confusa venta de una universidad privada, la Universidad Central, fueron algunos de los hechos que a principios de ese año movilizaron a los primeros contingentes de jóvenes. La cantidad de manifestantes fue aumentando paulatinamente gracias al errático accionar del gobierno en la búsqueda de soluciones a estas inquietudes y a que fueron incorporándose nuevas demandas a poco de

10 Entre los trabajos que refieren a esta temprana preocupación universitaria por la ciencia útil se puede consultar Diana Soto Arango, "El movimiento de estudiantes y catedráticos en Santa Fe de Bogotá a finales del siglo XVIII", en Movimientos estudiantiles en la historia de América Latina, Volumen I, coordinado por Renate Marsiske (Ciudad de México: Centro de Estudios sobre la Universidad de la Universidad Nacional Autónoma de México - Plaza y Valdés, 1999), 46. 
iniciado el conflicto, aquellas que desde comienzos de la década de 1990 venía realizando periódicamente el estudiantado antes de la cuenta pública anual del presidente del país, el 21 de mayo ${ }^{11}$. En el caso mexicano de 1968 sucedió algo parecido, en el sentido de que el problema que gatilló el movimiento puede ser comprendido como un entrevero menor: una pelea de barrio entre estudiantes de enseñanza secundaria que fue escalando gracias al desafortunado proceder de la policía en sus intentos por aplacarla ${ }^{12}$. En el movimiento brasileño de 1968 también se confirma lo dicho en la medida que fue la represión desmedida a una protesta causada por las malas condiciones que presentaba un comedor universitario, en Río de Janeiro, el hecho que estuvo en los orígenes del alzamiento $^{13}$. Mientras que en el movimiento argentino de 1918 lo dicho se refrenda al constatar que fue el rechazó estudiantil a una decisión de las autoridades de la Universidad de Córdoba de acabar con el internado en el Hospital de Clínicas, y de aumentar los requisitos académicos para los estudiantes de ingeniería, lo que estuvo en los orígenes del movimiento estudiantil ${ }^{14}$. Es oportuno reparar, aún a riesgo de parecer redundantes, que así como los hechos que detonaron a estos movimientos pueden ser juzgados como intrascendentes, las causas por detrás del surgimiento de los mismos tiene mucho que ver con la displicencia e incompetencia de las autoridades encargadas de atender las exigencias estudiantiles iniciales ${ }^{15}$.

Respecto de la masividad, de la cantidad de personas que se envolvió en la movilización chilena de 2011, se debe precisar que el número de manifestantes tendió a crecer sostenidamente desde abril de ese año hasta el domingo 21 de agosto, fecha en que se verificó su ápice al reunir a un millón de personas en un parque urbano de la capital ${ }^{16}$. Dato que cobra más espectacularidad si se repara que en ese entonces Santiago contaba con alrededor de seis millones de habitantes. La masividad del movimiento mexicano de 1968 se colige de datos como, por ejemplo, que hubo varias marchas multitudinarias que superaron ampliamente la matrícula total de universitarios del país, entre ellas la que el 27 de agosto congregó a cerca de cuatrocientas mil personas solo en la capital ${ }^{17}$. En Brasil, en tanto, el punto culmine del movimiento de 1968 fueron las marchas que en junio convocaron a decenas de miles de personas en Río de Janeiro. Marchas que adquieren más resonancia si se recuerda que para entonces el país estaba en medio de una dictadura y que esta ya se había cobrado la vida de estudiantes

11 Sobre las primeras demandas, las relativas a las becas y a la venta de la Universidad Central, puede consultarse Francisco Figueroa. Llegamos para quedarnos: crónicas de la revuelta estudiantil (Santiago de Chile: LOM, 2013), 52, 53, 67, 68; Giorgio Jackson op. cit. (2013), 19 y 64; y Juan Urra, "La movilización estudiantil chilena en 2011", Revista del Observatorio Social de América Latina, No. 31 (2012): 25 .

12 Ariel Rodríguez Kuri, “Los primeros días. Una explicación de los orígenes inmediatos del movimiento estudiantil de 1968”, Historia Mexicana, Vol. 53, No.1 (2003): 183.

13 Zuenir Ventura, 1968 o ano que não terminou (Rio de janeiro: Nova Fronteira, 1988), 97.

14 Pablo Buchbinder, Historia de las universidades argentinas (Buenos Aires: Editorial Sudamericana, 2010), 92 y 93.

15 Interpretación obtenida, entre otros textos, de José Auth y Federico Joannon, "El movimiento estudiantil: un marco conceptual", En El movimiento estudiantil: conceptos e historia, coordinado por Manuel Antonio Garretón y Javier (Santiago de Chile: Sur, 1985), 38.

16 Francisco Figueroa, op. cit., 146.

17 Gilberto Guevara Niebla, La libertad nunca se olvida: memoria del 68 (Ciudad de México: Cal y Arena, 2004), 117 y siguientes. 
movilizados $^{18}$. La masividad del caso argentino, en 1918, es bien ilustrada por el hecho de que aun cuando en Córdoba la matrícula universitaria bordeaba los dos mil estudiantes, fueron varias las concentraciones que superaron en más de diez veces dicho número ${ }^{19}$.

Sobre los actores que conformaron al movimiento chileno de 2011 se apunta que estos fueron diversos y, en general, se vinculaban al universo cultural. Esto tiene que ver con que junto al estudiantado universitario se movilizó también el estudiantado secundario, el profesorado primario, secundario y universitario, algunos profesionales jóvenes y una parte de la intelectualidad y del mundo artístico del país ${ }^{20}$. Se debe señalar, a su vez, que esta capacidad de aglutinar en torno a sí a amplios sectores del mundo cultural ha sido una constante en los movimientos estudiantiles descritos ${ }^{21}$. El caso chileno reseñado destaca por el protagonismo que tuvieron los estudiantes adscritos a instituciones de enseñanza privada ${ }^{22}$. El mexicano de 1968 porque contó con una participación decidida de profesores y de una buena parte de las autoridades universitarias ${ }^{23}$. El brasileño de 1968 porque alineó tras de sí a una fracción de la prensa nacional ${ }^{24}$. Y el argentino de 1918 porque concitó el apoyo de personalidades del mundo de la política ${ }^{25}$. Esta composición diversa de los movimientos, cruzada por la variable cultural, es la que explica la gran proliferación de demandas que ellos vehiculan y la centralidad que posee la educación en la solución de los problemas identificados. Y es que, como se adelantara, si en la época actual tanto los movimientos estudiantiles, en particular, como los movimientos sociales, en general, consiguen identificar los problemas sociales, políticos y económicos que constriñen a la sociedad, son los movimientos estudiantiles, en su calidad de fenómenos sociales con una fuerte impronta cultural, los que tienden a poner a la educación en el centro de sus propuestas o soluciones ${ }^{26}$.

$\mathrm{Al}$ analizar las formas mediante las cuales se movilizó el estudiantado chileno en 2011 se observa que ellas fueron las mismas que desde hace al menos cien años vienen primando entre el estudiantado latinoamericano, a saber: las paralizaciones de actividades, las ocupaciones de dependencias educacionales y/o gubernamentales, las concentraciones masivas y las marchas multitudinarias ${ }^{27}$.

18 Luís Antonio Groppo, Uma onda mundial de revoltas: movimentos estudantis de 1968 (Piracicaba: Unimep, 2005), 95.

19 Véase, entre otros textos, Juan Carlos Portantiero, op. cit., 42,52.

20 Manuel Riesco, op. cit, 203; Camila Vallejo, op. cit., 105.

21 Una interpretación en línea con la expuesta se puede consultar en José Auth y Federico Joannon, op.cit., 32.

22 Jackson, op. cit. (2013), 68.

23 Véase, por ejemplo, la participación del rector de la Universidad Nacional Autónoma de México y el cuerpo directivo de la institución en Javier Barros Sierra, 1968 conversaciones con Gastón García Cantú (Ciudad de México: Siglo XXI), 1972.

24 El correo de la mañana y el Diario de la tarde fueron los más afines al movimiento estudiantil informa María Ribeiro do Valle, op. cit.

25 Además de la participación decisiva que tuvo el presidente del país Hipólito Yrigoyen, pueden apreciarse las contribuciones que hiciera Alfredo Palacios, destacado político e intelectual, en Alfredo Palacios, "La reforma universitaria y el problema económico" en La reforma universitaria: desafíos y perspectivas noventa años después, compilado por Emir Sader, Pablo Gentili y Hugo Aboites (Buenos Aires: Consejo Latinoamericano de Ciencias Sociales, 2008), 223-227.

26 Andrés Donoso Romo y Mía Dragnic García, "Hacia la universidad pública: aproximación a la importancia del movimiento estudiantil chileno de 2011 en perspectiva latinoamericana”, en Los desafíos de la universidad pública en América Latina y el Caribe, varios autores (Buenos Aires: Consejo Latinoamericano de Ciencias Sociales, 2015), 120.

27 José Auth y Federico Joannon complementan esta apreciación cuando en el accionar estudiantil distinguen las acciones 
La paralización de actividades fue una de las medidas que cundió entre el estudiantado chileno luego de que el presidente en su cuenta anual no tratara, ni siquiera tangencialmente, las demandas levantadas por los jóvenes universitarios. Para apoyar la idea de lo extendidas que han sido estas medidas de presión entre el estudiantado latinoamericano se apunta que en el caso mexicano de 1968, en materia de marchas, el movimiento estudiantil no conoció declive. Fueron cinco grandes marchas las que se verificaron solo en Ciudad de México, algunas de las cuales superaron, según ciertas estimaciones, las trescientas mil personas ${ }^{28}$. Por su parte en el caso brasileño de 1968, bien conocida fue la ocupación de la Facultad de Filosofía de la Universidad de San Pablo. Acción que se realizó durante el segundo semestre de ese año por los propios estudiantes, toma que terminó luego de que en un enfrentamiento que involucraba a universitarios, paramilitares y policías muriera un estudiante ${ }^{29}$. En el caso argentino, en tanto, se recuerda que el 9 de septiembre de 1918, en Córdoba, los estudiantes tomaron la rectoría de la universidad. Un hecho que a la larga terminó desequilibrando la balanza a favor de los estudiantes e hizo que el movimiento consiguiera parte importante de sus demandas ${ }^{30}$.

Tanto la magnitud que tuvo el movimiento estudiantil chileno de 2011 en general, como la capacidad que tuvo de aglutinar a personas de distintos orígenes en particular, ocurrió porque contó desde muy temprano con una organización representativa capaz de imprimirle legitimidad y sentido al accionar de los jóvenes $^{31}$. La Confederación Nacional de Estudiantes de Chile, organización que existe desde la década de 1980 pero que remonta sus raíces a la primera mitad del siglo XX, se conforma con los dirigentes estudiantiles de las universidades mixtas del país -aquellas que reciben dineros públicos- y ese 2011, debido a la fuerza de las circunstancias, incluyó también a algunos representantes de universidades privadas ${ }^{32}$. En el caso mexicano de 1968 el Consejo Nacional de Huelga jugó un papel más que destacado, formándose expresamente para dicha ocasión luego de recoger los mejores aprendizajes de aquellas organizaciones similares que llevaron a buen término sus movilizaciones en los años anteriores ${ }^{33}$.

instrumentales, aquellas que persiguen presionar a las autoridades para así satisfacer alcanzar las demandas estudiantiles, de las acciones expresivas, aquellas que procuran fortalecer la identidad y cohesión interna entre los estudiantes. Véase José Auth y Federico Joannon, op. cit., 55 y 56.

28 Carlos Monsiváis, El 68: la tradición de la resistencia (Ciudad de México: Era, 2008), 86.

29 Flávia de Angelis Santana, Movimento estudantil e ensino superior no Brasil: a reforma universitária no centro da luta política estudantil dos anos 60 (San Pablo: Tesis Universidade de São Paulo, 2014), 173.

30 Gabriel Del Mazo, La reforma universitaria. Tomo I: el movimiento argentino (Lima: Universidad Nacional Mayor de San Marcos, 1967), 31-33.

31 Tanto Álvaro Acevedo Tarazona como Mauricio Archila destacan que para el caso colombiano es precisamente la ausencia de una organización aglutinadora y fuertemente legitimada la que impidió que el descontento estudiantil de mediados del siglo XX adquiriera una magnitud y dimensión nacional. Véase Álvaro Acevedo Tarazona, "Memoria e historia del movimiento estudiantil en Colombia (1968)" en Movimientos estudiantiles en la historia de América Latina, volumen IV, coordinado por Renate Marsiske (Ciudad de México: Instituto de Investigaciones sobre la Universidad y la Educación de la Universidad Nacional Autónoma de México, 2015), 113 y 114; y Mauricio Archila, "El movimiento estudiantil en Colombia", Revista del Observatorio Social de América Latina, No. 31 (2012): 82.

32 Francisco Figueroa, op. cit., 49 y 50; Giorgio Jackson, op. cit. (2013), 73.

33 José René Rivas Ontiveros, La izquierda estudiantil en la UNAM: organizaciones, movilizaciones y liderazgos (1958-1972) (Ciudad de México: Editorial Miguel Ángel Porrúa - Universidad Nacional Autónoma de México, 2007), 601-603. 
En caso brasileño de 1968 la Unión Nacional de Estudiantes, presente desde la década de 1930, mantenía una gran capacidad de movilización pese a operar en ese entonces en la clandestinidad ${ }^{34}$. Mientras que en el caso argentino de 1918 fueron la Federación Universitaria de Córdoba y la Federación Universitaria Argentina, ambas fundadas ese mismo año en la primera fase del conflicto, las que tuvieron el papel más destacado en la conducción del movimiento ${ }^{35}$. Dentro de este mismo punto se debe advertir que, a diferencia de lo ocurrido en el resto de las experiencias contrastadas, en Chile no existieron sectores organizados de estudiantes que se opusieran al movimiento. Cuestión que hizo que las disputas en el seno de la Confederación tuvieran que ver, principalmente, con cómo lograr la adhesión de aquellos estudiantes que aún no se plegaban a las jornadas de protesta y/o con cómo conseguir el máximo de simpatía entre la población nacional. Las diferencias al interior de este órgano de dirección serían, por tanto, más de énfasis que de fondo. Por un lado estaban los reformistas, aquellos dirigentes vinculados a alguno de los partidos políticos con representación parlamentaria, como el Socialista o el Comunista, que insistían en asegurar las conquistas concretas conseguidas en el campo educacional. Y por otro lado estaban los revolucionarios, aquellos que participaban en algunas de las así llamadas agrupaciones de izquierda extra-parlamentaria y que apostaban a radicalizar lo más posible el movimiento con miras a poner en jaque al modelo neoliberal imperante o, al menos, a provocarle una fractura ${ }^{36}$. En tanto, en los otros tres movimientos analizados, el mexicano de 1968, el brasileño de 1968 y el argentino de 1918, sí hubo oposición estudiantil y esta se aglutinó en torno a organizaciones que decían defender valores y tradiciones católicas.

Por último, desde un prisma eminentemente sociológico se observa que tanto el movimiento chileno de 2011 como los otros que aquí se están considerando, compartieron un antecedente estructural que puede ser leído, también, como parte de sus causas más trascedentes. Todos fueron precedidos por un explosivo aumento de la matrícula universitaria y, consecuentemente, por una ampliación y "popularización" del espectro socioeconómico del estudiantado. En Chile un dato que ayuda a dimensionar este fenómeno es que mientras en 1980 la matrícula de la educación superior bordeaba los 120 mil estudiantes, en 2011 ella se acercaba al millón de estudiantes ${ }^{37}$. En México, en tanto, si en 1950 se contaba con 30 mil estudiantes de nivel superior, en 1970 esa cifra ascendía a 270 mil $^{38}$.

34 Maria de Lourdes Fávero, A UNE em tempos de autoritarismo (Rio de Janeiro: Editora da Universidade Federal do Rio de Janeiro, 1994), 17.

35 Pablo Buchbinder, op. cit. (2008), 125.

36 Francisco Figueroa, op. cit., 102-105. Para aproximarse a las orgánicas estudiantiles chilenas neoliberales, muy actuantes en los años de la dictadura, acudir, entre otros textos, a Pablo Toro Blanco, "La vida de los otros: la FECECH y su conflictiva relación con el movimiento estudiantil en la Universidad de Chile (1978-1984)", en Movimientos estudiantiles en la historia de América Latina, volumen $I V$, coordinado por Renate Marsiske (Ciudad de México: Instituto de Investigaciones sobre la Universidad y la Educación de la Universidad Nacional Autónoma de México, 2015), 189-222.

37 Mauricio Olavarría Gambi, “¿Gratuidad y equidad? Interrogantes claves en la política de educación superior”, en Ciudadanía en marcha: educación superior y movimiento estudiantil 2011, curso y lecciones de un conflicto, coordinado por Sergio González y Jorge Montealegre (Santiago de Chile: Editorial Universidad de Santiago de Chile, 2012), 84.

38 Huascar Taborga Torrico, Expansión y diversificación de la matrícula de la educación superior en México (Ciudad de México: Asociación Nacional de Universidades e Institutos de Educación Superior, 2003), 10. 
En Brasil, por su parte, los registros de 1960 indican 64 mil estudiantes mientras que los de 1968 ya hablan de $214 \mathrm{mil}^{39}$. Y en Argentina, asimismo, si en 1906 había casi dos mil estudiantes matriculados, en 1920 ellos superaban los $12 \mathrm{mil}^{40}$. Todas transformaciones cuantitativas que tensionaban tanto la infraestructura como las estructuras universitarias y que, como es sensato deducir, tenían correlato en nuevas exigencias o demandas estudiantiles.

\section{Las demandas del movimiento estudiantil chileno de 2011 en clave latinoamericana}

El presente apartado seguirá la misma lógica utilizada en el anterior, es decir, se caracterizarán las dos principales demandas del movimiento estudiantil chileno de 2011 y después de cada una se desarrollarán algunas consideraciones sobre lo extendida o lo profunda que estaba dicha exigencia en los otros movimientos estudiantiles estudiados.

Después de la cuenta pública que el presidente diera ese 21 de mayo de 2011, debido a la nula receptividad hacia las demandas que el estudiantado venía levantando -que iban desde el reajuste de las becas de alimentación hasta el aumento del financiamiento estatal a las universidades-, los manifestantes redoblaron sus protestas y agudizaron sus análisis. Es a partir de entonces, por tanto, que empezaron a tomar forma las dos exigencias que terminaron dando su sello al movimiento: que la enseñanza universitaria fuera gratuita y que se pusiera fin al lucro en las instituciones de educación superior ${ }^{41}$.

La demanda por gratuidad era apuntalada en una suma de antecedentes disímiles. Había quienes recordaban que hasta antes de la dictadura los costos de las universidades habían sido absorbidos casi en su totalidad por el Estado, por lo tanto no era una idea inaudita ni extemporánea. Es más, cuando algunos personeros públicos alegaban falta de capacidad económica por parte del Estado para asumir una responsabilidad de esta envergadura, parte de los estudiantes proponía, inclusive, modificaciones en el presupuesto público para permitir alcanzarla. Había quienes entendían, en tanto, que la educación era un derecho que debía ser garantizado por el Estado y que, por tal razón, era necesario implementar una fórmula que garantizara la gratuidad de la enseñanza en el nivel superior. Y había quienes defendían esta exigencia, también, sosteniendo que ella hacía posible que fueran los motivos estrictamente educacionales los que primaran a la hora de decidir quién permanecía y quién se iba del sistema educacional, y no los de tipo económico como hasta entonces venía siendo la regla ${ }^{42}$.

Es importante destacar que en América Latina la demanda por gratuidad posee larga data. De hecho en el caso del movimiento estudiantil brasileño de

\footnotetext{
39 João Roberto Martins Filho, Movimento estudantil e ditadura militar, 1964-1968 (Campinas: Papirus, 1987), 125.

40 Pablo Buchbinder, op. cit. (2010), 118.

41 Andrés Donoso Romo y Mía Dragnic García, op. cit., 137-138; y Francisco Figueroa, op. cit., 65.

42 Juan Urra, op. cit., 26. Una aproximación analítica a la noción de gratuidad se puede revisar en Fernando Atria, Derechos sociales $y$ educación: un nuevo paradigma de lo público (Santiago de Chile: LOM, 2014), 81-211.
} 
1968 esta exigencia tuvo un lugar importante, sobre todo después de que ella se viera amenazada con el golpe de Estado de 1964 y el viraje neoliberal que experimentó el país durante los primeros años de la dictadura ${ }^{43}$. En el caso del movimiento mexicano de 1968 la gratuidad no fue amenazada directamente, aunque sí se consigna que en 1967 hubo un movimiento estudiantil de carácter nacional, originado en el norte del país, que consiguió federalizar una escuela superior de agricultura privada tornándola, de esta manera, gratuita; y sí se registra que al final del movimiento circulaban rumores que aludían al riesgo de cerrar las universidades públicas del país por no justificarse su labores ante la ausencia de estudiantes ${ }^{44}$. En el argentino de 1918, en tanto, la gratuidad sí fue discutida por el estudiantado nacional, más específicamente en el Primer Congreso Nacional de Estudiantes Universitarios realizado en Córdoba en julio de ese año, pero finalmente no se lograron acuerdos en la materia por lo que no fue incorporada al petitorio. Siendo solo a partir de la década siguiente que esta exigencia comenzó a formar parte de sus reivindicaciones ${ }^{45}$.

La segunda gran demanda del movimiento chileno de 2011, poner fin al lucro en las instituciones educacionales, también fue constituyéndose con base en antecedentes diversos. Los razonamientos más utilizados para defender este requerimiento subrayaban que era impropio de una universidad manejarse como si fuera una actividad económica cualquiera, es decir, donde primara el ánimo de obtener el máximo de ganancias para sus propietarios. En la misma línea se señalaba que obtener utilidades era incompatible con objetivos de tipo cultural o educacional, coexistencia inviable puesto que la procura de beneficios económicos siempre se impondría a la satisfacción de criterios culturales ${ }^{46}$. Argumentos que se reforzaban recordando que en Chile el lucro en la educación era una actividad ilegal ${ }^{47}$ y sosteniendo, como lo hiciera el dirigente de la Pontificia Universidad Católica ese 2011, Giorgio Jackson, que el lucro no había "demostrado ningún beneficio en la educación" ${ }^{\prime 8}$.

Al momento de rastrear la profundidad de esta demanda en otros escenarios de América Latina se constata que, pese a que otros movimientos estudiantiles se habían opuesto también a procesos de privatización de la educación, como el caso del brasileño en la década de 1960 o del colombiano el mismo $2011^{49}$. Fue el movimiento chileno de 2011, por las mismas condicionantes históricas que han hecho de este país una de las lumbreras del neoliberalismo mundial, el que

43 Rodrigo Patto Sá Motta, As universidades e o regime militar (Rio de Janeiro: Zahar, 2014), 9.

44 Consúltese, entre otros textos, José René Rivas Ontiveros, op. cit., 504,603.

45 María Cristina Vera, "Reformas, contrarreformas y movimientos estudiantiles en la Universidad de Córdoba (1870-1936)" en Movimientos estudiantiles en la historia de América Latina, volumen III, coordinado por Renate Marsiske (Ciudad de México: Centro de Estudios sobre la Universidad de la Universidad Nacional Autónoma de México - Plaza y Valdés, 2006), 50.

46 Una aproximación analítica a la exigencia de poner "fin al lucro" puede encontrarse en Cristián Bellei, Mercado y privatización de la educación chilena (Santiago de Chile: LOM, 2015), 165-191.

47 Sobre la ilegalidad de la obtención de ganancias asociadas a actividades educacionales véase, especialmente, María Olivia Mönckeberg, El negocio de las universidades en Chile (Santiago de Chile: Random House Mondadori, 2007).

48 Giorgio Jackson. "Con Atria en la mochila", en La mala educación: ideas que inspiran al movimiento estudiantil en Chile, de Fernando Atria (Santiago de Chile: Catalonia, 2012), 17.

49 Mauricio Archila, op. cit., 94. 
con su exigencia por poner fin al lucro en las universidades apuntó, de manera certera, al corazón de este ordenamiento. Sí, porque, así como no es lo mismo defender la gratuidad en contextos donde este derecho persiste, como ocurrió en el movimiento estudiantil que se desarrollo en 1999-2000 en la Universidad Nacional Autónoma de México o los verificados en diferentes universidades públicas brasileñas a principios del siglo XXI, que exigirla donde ella no sigue en curso $^{50}$. Tampoco es lo mismo demandar gratuidad que acabar con el lucro en las universidades. $\mathrm{Y}$ es que mientras lo primero apunta a reformar el sistema de financiamiento de la educación superior, lo segundo aspira a revolucionar los horizontes del mismo ${ }^{51}$.

\section{Desenlace y logros del movimiento chileno de 2011 en clave latinoamericana}

Para cerrar esta aproximación a las constantes que han tenido los grandes movimientos estudiantiles de la época contemporánea de América Latina, se referirá brevemente al desenlace y las conquistas obtenidas por el levantamiento chileno de 2011. Materias que se expondrán utilizando la misma fórmula de las secciones anteriores, es decir, luego de observado el caso chileno se harán algunos alcances tomando como referencia los otros movimientos considerados. Para cerrar el artículo, conforme lo propuesto en la sección inicial, se hará una breve síntesis con los principales aspectos compartidos por los movimientos estudiantiles estudiados. Síntesis que se espera contribuya a alimentar futuros diálogos sobre lo mucho que han compartido las luchas estudiantiles en la región durante los últimos cien años y, al mismo tiempo, que instigue a futuros investigadores a que puedan profundizar, contrastar o criticar estas interpretaciones.

En septiembre de 2011 el movimiento estudiantil chileno comenzó un vertiginoso repliegue debido al desgaste que el paso de los meses provoca en todo movimiento social de grandes dimensiones, a la ampliación de las desavenencias políticas en el seno de la dirigencia estudiantil y al severo trastrocamiento que sufrió la agenda de los medios de comunicación tras la muerte traumática de un grupo de personalidades ligadas al mundo de la televisión ${ }^{52}$. Así, paulatinamente, las diferentes medidas de presión estudiantil desplegadas durante los meses anteriores comenzaron a perder poder de convocatoria y, para diciembre de ese mismo año, el movimiento ya había entrado en una nueva etapa de latencia.

Al observar el desenlace de los otros movimientos estudiantiles contrastados salta a la vista que no hay aspectos que se repitan en este punto. Mientras el movimiento chileno se apagó producto de un desgaste más o menos inorgánico de sus medidas de presión, el mexicano de 1968 se desbandó luego de la masacre

50 Para acercarse al movimiento mexicano reseñado y sus relaciones con el neoliberalismo apreciar Adrián Sotelo Villegas, Neoliberalismo y educación: la huelga en la UNAM a finales de siglo (Ciudad de México: Ediciones el Caballito, 2000). Con el mismo fin, pero para aproximarse a la situación brasileña vivida a comienzos del siglo XXI acudir a Silene de Moraes Freire. "Movimento estudantil no Brasil: lutas pasadas, desafíos presentes”, Revista de Historia de la Educación Latinoamericana, No. 11 (2008): 142-144.

51 Fernando Atria. La mala educación: ideas que inspiran al movimiento estudiantil en Chile (Santiago de Chile: Catalonia, 2012 ), 108.

52 Francisco Figueroa, op. cit., 157. 
que sufrieron a manos de uniformados, el brasileño de 1968 se desmovilizó luego de que el gobierno apresara a cientos de dirigentes reunidos en un congreso nacional de estudiantes y el argentino de 1918 se apagó luego de que se atendieran sus principales demandas, entre ellas la relativa contar con participación estudiantil en el gobierno universitario. Pese a la imposibilidad de identificar, por el momento, alguna suerte de patrón en el repliegue de los grandes movimientos estudiantiles observados, pareciera que fuera el momento del diálogo entre manifestantes e interpelados, o de la imposibilidad evidente del mismo, el hito que marcaría su clímax y, al mismo tiempo, el comienzo del retorno a la normalidad. Aunque claro, se debe tener en cuenta que el movimiento argentino de 1918 tensiona esta hipótesis en la medida que luego del primer momento de diálogo, cuando desde el gobierno de la nación se decide intervenir a la Universidad de Córdoba y se produce un retorno a la normalidad, el estudiantado se mantuvo en una actitud vigilante que redundará en un segundo momento de apogeo que será, a la postre, al que le sucederán sus principales conquistas. Dicho en clave teórica, cuando el estudiantado hace uso de lo que con Sidney Tarrow se comprende como la estructura de oportunidades políticas, cuando producto de sus alianzas y sus medidas de presión logra instalar sus demandas como problemas prioritarios a resolver por las autoridades competentes, ahí se ubicaría el punto de inflexión que daría paso a su desenlace ${ }^{53}$. Desenlace que, claro está, no en todos los casos es sinónimo de resolución de las exigencias estudiantiles.

En cuanto a los logros del movimiento chileno de 2011 sobresale el hecho de que consiguiera, por un lado, posicionar a la educación en el centro de incontables debates públicos y privados ${ }^{54}$, y que lograra, por otro lado, impactos duraderos tanto en la dimensión simbólica como en el plano material de la sociedad. Sobre sus desdoblamientos simbólicos se apunta, como han reparado muchos analistas, que el movimiento se constituyó en el primer cuestionamiento contundente al ordenamiento neoliberal impuesto en dictadura, contribuyendo así a desenmascarar las constricciones que afectaban a parte sustantiva de la población, ayudando a redibujar esa imagen que existía de una sociedad con desafíos pero sin grandes conflictos, y favoreciendo una toma de conciencia entre la población sobre que sus problemas no eran residuales, circunstanciales o excepcionales, sino mas bien consustanciales al funcionamiento del modelo ${ }^{55}$. Sobre lo material, en tanto, se aprecia que desde 2012 en adelante se viene verificando un aumento significativo del presupuesto público destinado a la educación -de hecho, como recuerda el economista Manuel Riesco, solo entre el año 2011 y 2012 en el presupuesto nacional la partida de educación aumentó en cinco puntos

53 Tarrow, Sidney. El poder en movimiento. Los movimientos sociales, la acción colectiva y la política (Madrid: Alianza editorial, 1997), 49.

54 Sergio Grez, "Chile 2012: el movimiento estudiantil en la encrucijada”, en Le Monde Diplomatique (Chile), enero-febrero (2012), 7; Giorgio Jackson, op. cit. (2012), 18; y Juan Urra, op. cit., 36.

55 Francisco Figueroa, op. cit., 18, 21; Giorgio Jackson, op. cit. (2013), 110; Alberto Mayol, No al lucro: de la crisis del modelo a la nueva era política (Santiago de Chile: Random House Mondadori, 2012), 14-15 y 205-206; Daniel Núñez, op. cit., 62 y 66; y Manuel Riesco, op. cit., 195. 
porcentuales -56 . Dineros que se vienen utilizando para costear parte de los intereses que cobran los bancos por los créditos que otorgan a los estudiantes para financiar sus estudios y que, a partir de este año 2016, también se usan para financiar las anualidades de los estudiantes universitarios de más bajos recursos ${ }^{57}$. Con todo, se debe dejar bien sentado que todas estas medidas tomadas hasta el momento, aunque significativas, no han conseguido afectar al ethos neoliberal del sistema de educación superior que tan contundentemente enjuiciaran los estudiantes. Pues nada se ha avanzado, por ejemplo, en desmontar los mecanismos de competencia e individualismo que priman entre agentes e instituciones y nada, tampoco, en lo que respecta a frenar el lucro en las universidades.

Cuando se observan los logros conseguidos por los otros grandes movimientos estudiantiles cotejados es posible apreciar aspectos compartidos. Esto en el sentido de que ninguno muestra victorias o derrotas categóricas, muestran mas bien avances en algunos aspectos fundamentales unidos a derrotas que no ofrecen espacio a doble lectura. Asimismo resalta que todos los movimientos, al ser situados en un marco temporal más amplio, dan pie a interpretaciones que no siempre se condicen con las evaluaciones que se generaron al calor de los acontecimientos. Por ejemplo, no cabe duda que el movimiento mexicano de 1968, debido a la masacre que sellaría su suerte, fue una derrota incontestable. Pero tampoco cabe duda que, como lo han hecho notar algunos analistas, puede comprenderse como el primer referente de una persistente lucha por la democracia que se proyecta hasta nuestros días ${ }^{58}$. Una lectura similar, aunque un poco menos categórica, es la que puede hacerse de las conquistas del movimiento estudiantil brasileño de 1968. Esto en razón de que aún cuando este haya sido claramente desarticulado luego del encarcelamiento de prácticamente la totalidad de su dirigencia, al tiempo que la dictadura recrudecía aún más su autoritarismo, son muchos también los que con el paso de los años tendieron a juzgarlo como fundamental en la recuperación de la democracia que empezó a hacerse tangible a fines de la década siguiente ${ }^{59}$. El caso argentino de 1918, en tanto, reafirma lo dicho pero desde un ángulo contrario, pues aún cuando exista un amplio consenso respecto de lo exitoso que fue al lograr concretar su principal demanda, la historiografía también enseña que diez años más tarde la situación en la Universidad de Córdoba se había revertido hasta el punto de anular todas las conquistas precedentes ${ }^{60}$.

56 Manuel Riesco, op. cit., 202.

57 Para más información sobre la nueva arquitectura de financiamiento público a la educación superior consultar, entre otras fuentes, www.gratuidad.cl

58 Allier Montaño, Eugenia. "Presentes-pasados del 68 mexicano. Una historización de las memorias públicas del movimiento estudiantil, 1968-2007”. Revista Mexicana de Sociología, Vol. 71, No. 2 (2009): 289.

59 Angélica Müller, A resistência do movimento estudantil brasileiro contra o regime ditatorial e o retorno da UNE à cena pública (19691979) (São Paulo: Tesis Universidade de São Paulo, 2010): 31.

60 Roberto Ferrero, op. cit., 41. 


\section{CONCLUSIÓN}

Anotados los desenlaces y logros del movimiento estudiantil chileno de 2011, y contrastados con lo sucedido en esta materia en los otros levantamientos estudiados, se procede a esbozar las constantes que se han identificado a lo largo del texto. Primero, se aprecia que los grandes movimientos estudiantiles han sido detonados por hechos que pueden ser juzgados como problemas menores mal gestionados por las autoridades competentes. Segundo, se observa que estos grandes movimientos conjugan demandas de tipo gremial o educacional junto a exigencias de tinte político o social. Tercero, se repara en que estos movimientos han sido conformados por una diversidad de actores, todos ligados en alguna medida al mundo cultural. Cuarto, se constata que las formas en que el estudiantado se ha movilizado no han variado sustantivamente, siendo la paralización de actividades, la ocupación de instituciones educacionales, las marchas por puntos claves de la ciudad y las grandes concentraciones públicas algunas de las más características. Quinto, se da cuenta que estos fenómenos han estado liderados por organizaciones autónomas que han contado con gran legitimidad y que, por lo mismo, han contado con gran poder de convocatoria. Sexto, se percibe que más allá de las razones coyunturales, circunstanciales o estructurales por detrás de estos movimientos, ellos han sido precedidos por un aumento brusco de la matrícula, fenómeno que probablemente es el que está detrás de los malestares que los animan y de las novedosas exigencias que en ellos aparecen. Séptimo, se advierte que la demanda por gratuidad levantada por el movimiento chileno de 2011 posee raíces centenarias en el contexto latinoamericano, esto en el sentido que ha estado presente, aunque con un protagonismo dispar, desde el movimiento de Córdoba en 1918. Y octavo, se aprecia que la exigencia por poner fin al lucro, aunque cuenta con vastos precedentes tanto en Chile como en América Latina, es la primera vez que adquiere tal centralidad y se vaticina que ella continuará siendo enarbolada por futuros movimientos estudiantiles, sobre todo cuando se vayan haciendo patente las debilidades del ordenamiento neoliberal en educación superior, entre ellas su propensión a generar varios circuitos educacionales, con diferencias notorias en cuanto a calidad, en función de la capacidad de pago y/o endeudamiento del estudiantado.

\section{REFERENCIAS}

Acevedo Tarazona, Álvaro. “Memoria e historia del movimiento estudiantil en Colombia (1968)". En Movimientos estudiantiles en la historia de América Latina, volumen IV, coordinado por Renate Marsiske. Ciudad de México: Instituto de Investigaciones sobre la Universidad y la Educación de la Universidad Nacional Autónoma de México, 2015, 109-127. 
Allier Montaño, Eugenia. “Presentes-pasados del 68 mexicano. Una historización de las memorias públicas del movimiento estudiantil, 1968-2007". Revista Mexicana de Sociología, Vol. 71, No. 2 (2009): 287-317.

Archila, Mauricio. "El movimiento estudiantil en Colombia". Revista del Observatorio Social de América Latina, No. 31 (2012): 71-103.

Atria, Fernando. La mala educación: ideas que inspiran al movimiento estudiantil en Chile. Santiago de Chile: Catalonia, 2012.

Atria, Fernando. Derechos sociales y educación: un nuevo paradigma de lo público. Santiago de Chile: LOM, 2014.

Auth, José y Joannon, Federico. "El movimiento estudiantil: un marco conceptual". En El movimiento estudiantil: conceptos e historia, coordinado por Manuel Antonio Garretón y Javier Martínez. Santiago de Chile: Sur, 1985, 13-58.

Barros Sierra, Javier. 1968 conversaciones con Gastón García Cantú. Ciudad de México: Siglo XXI, 1972.

Bellei, Cristián. Mercado y privatización de la educación chilena. Santiago de Chile: LOM, 2015.

Buchbinder, Pablo. ¿Revolución en los claustros? La Reforma Universitaria de 1918. Buenos Aires: Editorial Sudamericana, 2008.

Buchbinder, Pablo. Historia de las universidades argentinas. Buenos Aires: Sudamericana, 2010 [2005].

Ciria, Alberto y Sanguinetti, Horacio. La reforma universitaria (1918-2006). Santa Fe: Universidad Nacional del Litoral, 2006.

Castillo Troncoso, Alberto del. Ensayo sobre el movimiento estudiantil de 1968: la fotografía y la construcción de un imaginario. Ciudad de México: Instituto Mora - Instituto de Investigaciones sobre la Universidad y la Educación de la Universidad Nacional Autónoma de México, 2012.

Donoso Romo, Andrés y Dragnic García, Mía. “Hacia la universidad pública: aproximación a la importancia del movimiento estudiantil chileno de 2011 en perspectiva latinoamericana". En: Varios Autores. Los desafíos de la universidad pública en América Latina y el Caribe, Buenos Aires: Consejo Latinoamericano de Ciencias Sociales, 2015, pp. 119-145.

Donoso Romo, Andrés. Identidad y educación en América Latina. Ensayos, Caracas: Laboratorio Educativo, 2012.

Fávero, María de Lourdes. A UNE em tempos de autoritarismo. Rio de Janeiro: Editora da Universidade Federal do Rio de Janeiro, 1994.

Fernández Retamar, Roberto. Pensamiento de Nuestra América. Buenos Aires: Consejo Latinoamericano de Ciencias Sociales, 2006.

Ferrero, Roberto. Historia crítica del movimiento estudiantil de Córdoba, Tomo 1 (1918-1943). Córdoba: Alción, 1999.

Figueroa, Francisco. Llegamos para quedarnos: crónicas de la revuelta estudiantil. Santiago de Chile: LOM, 2013. 
Freire, Silene de Moraes. "Movimento estudantil no Brasil: lutas pasadas, desafíos presentes", Revista de Historia de la Educación Latinoamericana, No. 11 (2008): 131-146.

González Casanova, Pablo. Imperialismo y liberación: una introducción a la historia contemporánea de América Latina. Ciudad de México: Siglo XXI, 1985 [1979].

Grez, Sergio. “Chile 2012: el movimiento estudiantil en la encrucijada”. Le Monde Diplomatique (Chile), enero-febrero (2012): 7-8.

Groppo, Luís Antonio. Uma onda mundial de revoltas: movimentos estudantis de 1968. Piracicaba: Unimep, 2005.

Guevara Niebla, Gilberto. La libertad nunca se olvida: memoria del 68. Ciudad de México: Cal y Arena, 2004.

Jackson, Giorgio. "Con Atria en la mochila". En La mala educación: ideas que inspiran al movimiento estudiantil en Chile, de Fernando Atria. Santiago de Chile: Catalonia, 2012, pp. 13-19.

Jackson, Giorgio. El país que soñamos. Santiago de Chile: Random House Mondadori, 2013.

Marsiske, Renate. Movimientos estudiantiles en América Latina: Argentina, Perú, Cuba y México, 1918-1929. Ciudad de México: Centro de Estudios sobre la Universidad - Universidad Nacional Autónoma de México, 1989.

Martins Filho, João Roberto. Movimento estudantil e ditadura militar, 1964-1968. Campinas: Papirus, 1987.

Mayol, Alberto. No al lucro: de la crisis del modelo a la nueva era política. Santiago de Chile: Random House Mondadori, 2012.

Mazo, Gabriel del. La reforma universitaria. Tomo I: el movimiento argentino. Lima: Universidad Nacional Mayor de San Marcos, 1967.

Mönckeberg, María Olivia. El negocio de las universidades en Chile. Santiago de Chile: Random House Mondadori, 2007.

Monsiváis, Carlos. El 68: la tradición de la resistencia. Ciudad de México: Era, 2008.

Motta, Rodrigo Patto Sá. As universidades e o regime militar. Rio de Janeiro: Zahar, 2014.

Müller, Angélica. A resistência do movimento estudantil brasileiro contra o regime ditatorial e o retorno da UNE à cena pública (1969-1979). São Paulo: Tesis Universidade de São Paulo, 2010.

Núñez, Daniel. "Proyecciones políticas del movimiento social por la educación en Chile". Revista del Observatorio Social de América Latina, No. 31, (2012): 61-70.

Olavarría Gambi, Mauricio. “¿Gratuidad y equidad? Interrogantes claves en la política de educación superior". En Ciudadanía en marcha: educación superior y movimiento estudiantil 2011, curso y lecciones de un conflicto, coordinado por Sergio González y Jorge Montealegre. Santiago de Chile: Editorial Universidad de Santiago de Chile, 2012, 81-94.

Palacios, Alfredo. "La reforma universitaria y el problema económico". En La reforma universitaria: desafíos y perspectivas noventa años después, compilado por Emir Sader, Pablo Gentili y Hugo Aboites. Buenos Aires: Consejo Latinoamericano de Ciencias Sociales, 2008 [1928], 223-227. 
Poerner, Artur José. O poder jovem. História da participação política dos estudantes brasileiros. San Pablo: Centro de Memória da Juventude, 1995 [1968].

Portantiero, Juan Carlos. Estudiantes y política en América Latina: el proceso de la reforma universitaria (1918-1930). Ciudad de México: Siglo XXI, 1987 [1978].

Ramírez, Ramón. El movimiento estudiantil de México (julio/diciembre de 1968). Ciudad de México: Era y Benemérita Universidad Autónoma de Puebla, 2008 [1969].

Riesco, Manuel. Parto de un siglo: una mirada al mundo desde la izquierda de América Latina. Santiago de Chile: Centro de Estudios Nacionales de Desarrollo Alternativo Editorial Universidad de Santiago de Chile, 2012.

Ribeiro do Valle, María. 1968, o diálogo é a violência: movimento estudantil e ditadura militar no Brasil. Campinas: Editora Unicamp, 2010 [1999].

Rivas Ontiveros, José René. La izquierda estudiantil en la UNAM: organizaciones, movilizaciones y liderazgos (1958-1972). Ciudad de México: Editorial Miguel Ángel Porrúa Universidad Nacional Autónoma de México, 2007.

Rodríguez Kuri, Ariel. “Los primeros días. Una explicación de los orígenes inmediatos del movimiento estudiantil de 1968". Historia Mexicana, Vol. 53, n¹ (2003): 179-228.

Sanfelice, José Luis. Movimento estudantil, a UNE na resistência ao golpe de 1964. Campinas: Editora Alínea, 2008 [1986].

Santana, Flávia de Angelis. Movimento estudantile ensino superior no Brasil: a reforma universitária no centro da luta política estudantil dos anos 60. San Pablo: Tesis Universidade de São Paulo, 2014.

Sotelo Villegas, Adrián. Neoliberalismo y educación: la huelga en la UNAM a finales de siglo. Ciudad de México: Ediciones el Caballito, 2000.

Soto Arango, Diana. “El movimiento de estudiantes y catedráticos en Santa Fe de Bogotá a finales del siglo XVIII". En Movimientos estudiantiles en la historia de América Latina, Volumen I, coordinado por Renate Marsiske. Ciudad de México: Centro de Estudios sobre la Universidad de la Universidad Nacional Autónoma México - Plaza y Valdés, 1999, 40-60.

Taborga Torrico, Huáscar. Expansión y diversificación de la matrícula de la educación superior en México. Ciudad de México: Asociación Nacional de Universidades e Institutos de Educación Superior, 2003.

Tarrow, Sidney. El poder en movimiento. Los movimientos sociales, la acción colectiva y la política. Madrid: Alianza editorial, 1997.

Toro Blanco, Pablo. “La vida de los otros: la FECECH y su conflictiva relación con el movimiento estudiantil en la Universidad de Chile (1978-1984)". En Movimientos estudiantiles en la historia de América Latina, volumen IV, coordinado por Renate Marsiske. Ciudad de México: Instituto de Investigaciones sobre la Universidad y la Educación de la Universidad Nacional Autónoma de México, 2015, 189-222.

Urra, Juan. "La movilización estudiantil chilena en 2011". Revista del Observatorio Social de América Latina, No. 31 (2012): 23-37.

Vallejo, Camila. Podemos cambiar el mundo. Santiago de Chile: Ocean Sur, 2012. 
Ventura, Zuenir. 1968 o ano que não terminou. Rio de janeiro: Nova Fronteira, 1988.

Vera, María Cristina. "Reformas, contrarreformas y movimientos estudiantiles en la Universidad de Córdoba (1870-1936)”. En Movimientos estudiantiles en la historia de América Latina, volumen III, coordinado por Renate Marsiske. Ciudad de México: Centro de Estudios sobre la Universidad - Universidad Nacional Autónoma México - Plaza y Valdés, 2006.

Zermeño, Sergio. México una democracia utópica: el movimiento estudiantil del 68. Ciudad de México: Siglo XXI, 2010.

\begin{tabular}{l|l|} 
& \\
\hline $\begin{array}{l}\text { Donoso Romo, Andrés. “Constantes en los movimientos } \\
\text { estudiantiles latinoamericanos: Aproximación a partir del caso } \\
\text { chileno de 2011". Revista Historia de la Educación Latinoamericana. } \\
\text { Vol. } 19 \text { No. } 28 \text { (2017): 71-90 }\end{array}$ & \\
\hline
\end{tabular}

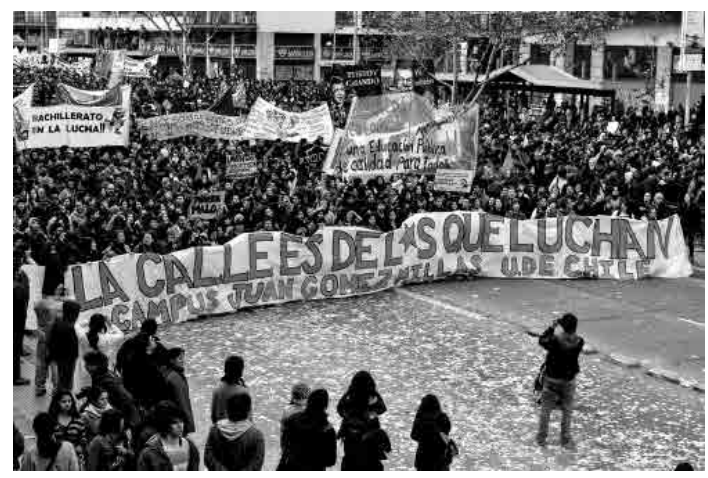

Fuente: https://www.tumblr.com/search/movimiento\%20estudiantil\%20chile\%202011 